\title{
Power Transformer Fault Diagnosis Using Fuzzy Reasoning Spiking Neural P Systems
}

\author{
Yousif Yahya', Ai Qian1, Adel Yahya² \\ ${ }^{1}$ Department of Electrical Engineering, Shanghai Jiao Tong University, Shanghai, China \\ ${ }^{2}$ Department of Industrial Engineering, Al-Zawia University, Regdalen, Libya \\ Email: yousif77@sjtu.edu.cn, yousifadel11@gmail.com
}

How to cite this paper: Yahya, Y., Qian, A. and Yahya, A. (2016) Power Transformer Fault Diagnosis Using Fuzzy Reasoning Spiking Neural P Systems. Journal of Intelligent Learning Systems and Applications, 8, 77-91.

http://dx.doi.org/10.4236/jilsa.2016.84007

Received: August 15, 2016

Accepted: November 8, 2016

Published: November 11, 2016

Copyright $\odot 2016$ by authors and Scientific Research Publishing Inc. This work is licensed under the Creative Commons Attribution International License (CC BY 4.0).

http://creativecommons.org/licenses/by/4.0/

\begin{abstract}
This paper presents an intelligent technique to fault diagnosis of power transformers dissolved and free gas analysis (DGA). Fuzzy Reasoning Spiking neural P systems (FRSN P systems) as a membrane computing with distributed parallel computing model is powerful and suitable graphical approach model in fuzzy diagnosis knowledge. In a sense this feature is required for establishing the power transformers faults identifications and capturing knowledge implicitly during the learning stage, using linguistic variables, membership functions with "low", "medium", and "high" descriptions for each gas signature, and inference rule base. Membership functions are used to translate judgments into numerical expression by fuzzy numbers. The performance method is analyzed in terms for four gas ratio (IEC 60599) signature as input data of FRSN P systems. Test case results evaluate that the proposals method for power transformer fault diagnosis can significantly improve the diagnosis accuracy power transformer.
\end{abstract}

\section{Keywords}

Dissolved Gas Analysis, Fault Diagnosis, Fuzzy Reasoning,

Power Transformer Faults, Spiking Neural P System

\section{Introduction}

Today the electric networks become larger and more complex with big data received from a lot of events in different sections, among which power transformer is one of the most important sections in power systems. Any fault in the transformer can cause a severe outage, which therefore necessitates continuous monitoring and diagnostics of its operation. In this sense, any faults caused in power transformers will produce a lot of alarms, some of which are uncertain, incomplete and misinformed, thus, it is necessary 
to develop a good method to help dispatchers evaluate where the faults are and which transformer fail. However transformer fault diagnosis decision-making based on dissolved and free gas analysis (DGA) diagnostic methods may give conflict analysis results and complicate the final decision making by operators [1].

In fact, intelligent fault diagnosis systems are necessary to deal with changes in typology of power network to fast diagnose the fault stat and location of power transformers faults [2].

In recent years, artificial intelligence approaches have been proposed with high performance programs and in developing more smart diagnostic techniques for power transformers based on DGA methods, such as support vector machine [1] [3] [4] [5], fuzzy logic [6] [7] [8] [9] [10], neural network [11]-[18], grey clustering [19] [20], wavelet networks [21].

However, these approaches are using several techniques for detecting transformer faults based gases concentrations in the oil and DGA is recognized as the most informative method. This method involves sampling the oil and testing the sample to measure the concentration of the dissolved gases. The standards are associated with sampling, testing, and analyzing the results such as the standard IEC 60599 [22].

As a newly attractive research field of computer science, fuzzy reasoning spiking neural P systems (FRSN P systems), formally introduced by Hong Peng 2013 [23], which are a class of SN P systems with distributed and parallel computing models.

In this paper, FRSN P systems are introduced as diagnostic technique to tackle the power transformer faults based on DGA, and can be viewed as a directed graph; reasoning steps and transmits pulses from input proposition neurons to the output proposition neurons under the control of firing/spiking mechanism of neurons [24].

Furthermore, this method uses the IEC ratio gases as input signature to FRSN P systems diagnosis model to establish the fault reasoning results with confidence levels, based on confidence levels for different fault types of transformer can get decision which one faulty. In addition, fault diagnosis process is expressed by assume the initial parameters of FRSN P systems model with linguistic terms to give operators more accuracy to describe the degree of uncertainty fault information [25].

This paper is organized as follows. Section 2 provides the definitions of FRSN P systems. Section 3 presents power transformer DGA based on FRSN P systems and fault diagnosis model. Section 4 discusses the test results. Finally, conclusions and proposals for future work are given in Section 5 .

\section{Fuzzy Reasoning Spiking Neural P Systems}

\subsection{Definition of Fuzzy Reasoning Spiking Neural P System}

A FRSN P system with degree $m \geq 1$ is a construct of the form [23];

$$
\Pi=\left(A, \sigma_{1}, \cdots, \sigma_{m}, \text { syn }, I, O\right)
$$

where:

1. $A=\{a\}$ is a spike in the neurons;

2. $\sigma_{p}^{1}, \cdots, \sigma_{p}^{n}$ are proposition, $\sigma_{r}^{1}, \cdots, \sigma_{r}^{u}$ rule neurons and $m=n+u$ of the form; 


$$
\sigma_{i}=\left(\alpha_{i}, \tau_{i}, r_{i}\right), 1 \leq i \leq m
$$

where:

A. $\alpha_{i}$ is spikes potential value of neuron $\sigma_{i}$ expressed by $[0,1]$;

B. $\tau_{i}$ is truth value of neuron $\sigma_{i}$ expressed by $[0,1]$;

C. $r_{i}$ is a firing rule of neuron $\sigma_{i}$ of the form $E / a^{\alpha} \rightarrow a^{\beta}$ where:

a) $E$ is a regular expression.

b) $\alpha \& \beta$ are expressed by $[0,1]$.

3. $s y n$ is a directed graph of synapses between neurons, where:

$$
\text { syn } \subseteq\{1,2, \cdots, m\} \times\{1,2, \cdots, m\} \text {, with } i \neq j \text { for all }(i, j) \in \operatorname{syn}, 1 \leq i, j \leq m .
$$

4. $I, O \in\{1,2, \cdots, m\}$ are input and output neuron respectively.

\subsection{FRSN P Systems with Fuzzy Production Rules}

According to their usage in this study, neurons in FRSN P systems are classified into four types of neurons;

\section{Proposition neurons}

In this kind of neuron, If neuron as input proposition neuron in $\Pi$, then $\alpha=\tau$; otherwise $\alpha$ equals all pulse values received from their presynaptic rule neurons based on logical or operation [23].

\section{General rule neurons}

If neuron as general rule neuron in $\Pi$, then the pulse value equals the pulse value received from their presynaptic proposition neuron [2], their representation by fuzzy production rules;

$$
R_{i}\left(c_{i}\right): P_{j}\left(\alpha_{j}\right) \rightarrow P_{k}\left(\alpha_{k}\right)
$$

As shown in Figure 1(a), The fuzzy truth value of the of proposition $P_{k}$ is $\alpha_{k}=\alpha_{j} * c_{i}$.

\section{And rule neurons}

If neuron as and rule neuron in $\Pi$, then the pulse value equals all pulse values received from their presynaptic proposition neurons based on logical and operation [23], their representation by fuzzy production rules;

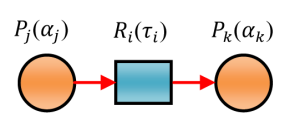

(a)

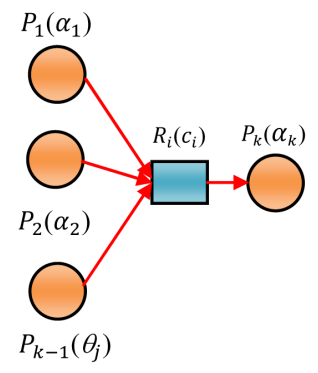

(b)

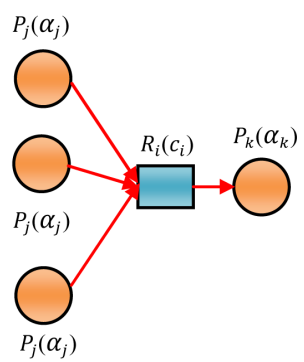

(c)

Figure 1. FRSN P systems rule neurons. (a) A general rule neuron, $\alpha_{k}=\alpha_{j} * \tau_{i}$; (b) And rule neuron, $\alpha_{k}=\min \left\{\alpha_{1}, \alpha_{2}, \cdots, \alpha_{k-1}\right\} * \tau_{i}$; (c) Or rule neuron, $\alpha_{k}=\max \left\{\alpha_{1}, \alpha_{2}, \cdots, \alpha_{k-1}\right\} * \tau_{i}$. 


$$
R_{i}\left(c_{i}\right): P_{1}\left(\alpha_{1}\right) \wedge P_{2}\left(\alpha_{2}\right) \wedge \cdots \wedge P_{k-1}\left(\alpha_{k-1}\right) \rightarrow P_{k}\left(\alpha_{k}\right)
$$

As shown in Figure 1(b), The fuzzy truth value of propositions $P_{k}$ is $\alpha_{k}=\min \left\{\alpha_{1}, \alpha_{2}, \cdots, \alpha_{k-1}\right\} * c_{i}$.

4. Or rule neurons

If neuron as or rule neuron in $\Pi$, then the pulse value equals all pulse values received from their presynaptic proposition neurons based on logical or operation [23], their representation by fuzzy production rules;

$$
R_{i}\left(c_{i}\right): P_{1}\left(\alpha_{1}\right) \vee P_{2}\left(\alpha_{2}\right) \vee \cdots \vee P_{k-1}\left(\alpha_{k-1}\right) \rightarrow P_{k}\left(\alpha_{k}\right)
$$

As shown in Figure 1(c), The fuzzy truth value of the of proposition $P_{k}$ is $\alpha_{k}=\max \left\{\alpha_{1}, \alpha_{2}, \cdots, \alpha_{k-1}\right\} * c_{i}$.

\subsection{Reasoning Matrix with Execution Rules}

We defined some matrices, reasoning processes and execution rules as follows [23].

1) $\alpha_{p}=\left(\alpha_{p}^{1}, \alpha_{p}^{2}, \cdots, \alpha_{p}^{n}\right)^{\mathrm{T}}$ is a fuzzy truth value vector of $\mathrm{n}$ proposition neurons.

2) $\alpha_{r}=\left(\alpha_{r}^{1}, \alpha_{r}^{2}, \cdots, \alpha_{r}^{u}\right)^{\mathrm{T}}$ is a fuzzy truth value vector of the u rule neurons.

3) $C=\operatorname{diag}\left(\tau_{1}, \tau_{2}, \cdots, \tau_{u}\right)$ is diagonal matrix confidence factor of rule neurons.

4) $S_{P \rightarrow G}=\left(s_{i j}\right)_{n \times u}, S_{P \rightarrow \wedge}=\left(s_{i j}\right)_{n \times u}, S_{P \rightarrow \vee}=\left(s_{i j}\right)_{n \times u}$ is directed synaptic matrix from proposition to general, and and or rule neurons respectively.

5) $S_{R \rightarrow P}=\left(s_{j i}\right)_{u \times n}$ is directed synaptic matrix from rule to proposition neurons.

In order to represent the execution rules of FRSN P systems formally, we introduce some fuzzy matrix operations [23].

1) $\oplus: C=A \oplus B$, where $A, B$ and $C$ are all $r \times s$ matrices, $c_{i j}=\max \left\{a_{i j}, b_{i j}\right\}$.

2) $\otimes: C=A \oplus B$, where $A, B$ and $C$ are all $r \times s, s \times t$ and $r \times t$ matrices respectively, $c_{i j}=\max _{1 \leq r \leq s}\left\{a_{i r}, b_{r j}\right\}$.

3) $\odot: C=A \oplus B$, where $A, B$ and $C$ are all $r \times s, s \times t$ and $r \times t$ matrices respectively, $c_{i j}=\min _{1 \leq r \leq s}\left\{a_{i r}, b_{r j}\right\}$.

\section{FRSN P Systems Fault Diagnosis Based on DGA}

\subsection{Transformer Fault Diagnosis Dissolved Gas Analysis}

Dissolved gas analysis (DGA) is powerful technique has been used to identify the incipient power oil transformers faults. In this technique can be identified according to the gases concentrations dissolved in oil of transformer, hydrogen $\left(\mathrm{H}_{2}\right),\left(\mathrm{CH}_{4}\right),\left(\mathrm{C}_{2} \mathrm{H}_{6}\right)$, $\left(\mathrm{C}_{2} \mathrm{H}_{4}\right),\left(\mathrm{C}_{2} \mathrm{H}_{2}\right)$, various interpretative DGA methods has been established, such as Gas key method, IEC ratio method, and the graphical representation method [1].

In this study we propose adaptive IEC ratio (AIEC ratio) method as first incipient diagnosis of the possible faults of oil transformer, in order to identifying the fault types based incipient possible faults diagnosed by IEC ratio method, we use the ratio of gases as input data to FRSN P systems diagnosis model and the output fuzzy reasoning results as shown in Figure 2. 


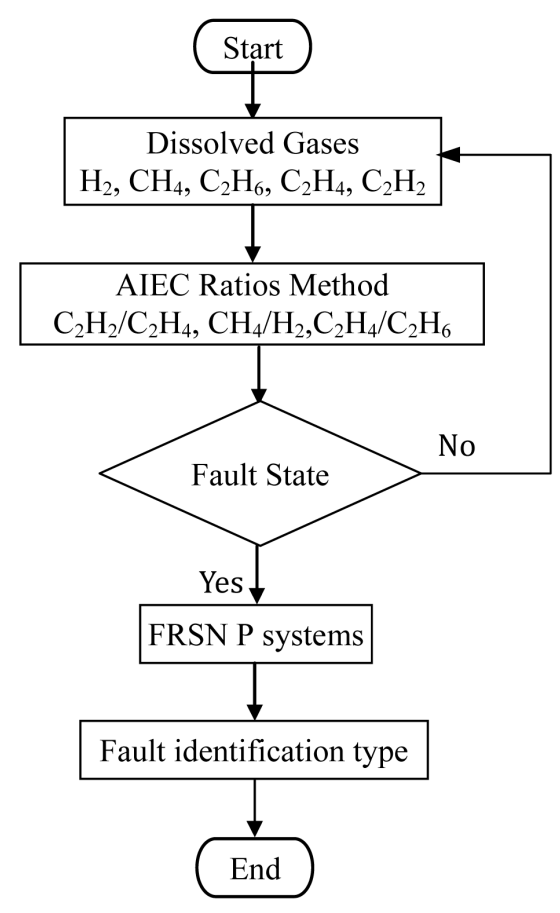

Figure 2. Transformer fault diagnosis based on DGA \& FRSN P systems.

\subsection{IEC Ratio Method}

In IEC ratio method, five gases, $\mathrm{H}_{2}, \mathrm{CH}_{4}, \mathrm{C}_{2} \mathrm{H}_{2}, \mathrm{C}_{2} \mathrm{H}_{4}$ and $\mathrm{C}_{2} \mathrm{H}_{6}$, as concentration gases in oil transformer. From these gases produce three ratios [21];

$$
R_{1}=\left(\mathrm{C}_{2} \mathrm{H}_{2} / \mathrm{C}_{2} \mathrm{H}_{4}\right), R_{2}=\left(\mathrm{CH}_{4} / \mathrm{H}_{2}\right), R_{3}=\left(\mathrm{C}_{2} \mathrm{H}_{4} / \mathrm{C}_{2} \mathrm{H}_{6}\right)
$$

Table 1 presents the transformer DGA faults are classified to six types, low energy discharge, high energy discharge, partial discharge, low thermal, medium thermal and high thermal faults, which is widely used to interpret the DGA [1].

Table 2 shows the interpreting fault types of IEC 60599 standard with values of three gas ratio $R_{1}, R_{2}, R_{3}$ [22].

\subsection{Adaptive IEC Ratio with Fuzzy Representation}

From the operator expert knowledge, in real word fault diagnosis events, in this study linguistic terms are always used to express the fault types related with gas concentrations ratio, such as $\left(\mathrm{C}_{2} \mathrm{H}_{2}\right) /\left(\mathrm{C}_{2} \mathrm{H}_{4}\right)$ very low, low, medium, high and very high in the transformer oil.

In this proposed method, we use the linguistic terms to describe a degree of gas concentrations ratio to become more capable to use fuzzy knowledge with fuzzy numbers. We can use adaptive IEC ratio (AIEC ratio) to deal with FRSN P systems and graphically represents with fault diagnosis model from input proposition neurons by reasoning steps to reach the final rezoning results after computation halts in output proposition neurons.

Table 3 shows the classification of gas ratio concentration based on IEC 60599 Gas ratio Limits in Table 2. 
Table 1. Fault types interpretationof DGA.

\begin{tabular}{cc}
\hline Fault type Characteristic & Code \\
Low energy discharge & $\mathrm{D}_{1}$ \\
High energy discharge & $\mathrm{D}_{2}$ \\
Partial discharge & $\mathrm{PD}$ \\
Thermal faults $T<300^{\circ} \mathrm{C}$ & $\mathrm{T}_{1}$ \\
Thermal faults $300^{\circ} \mathrm{C}<T<700^{\circ} \mathrm{C}$ & $\mathrm{T}_{2}$ \\
Thermal faults $T>700^{\circ} \mathrm{C}$ & $\mathrm{T}_{3}$ \\
\hline
\end{tabular}

Table 2. IEC60599 gas ratio limits.

\begin{tabular}{|c|c|c|c|}
\hline $\begin{array}{c}R_{1} \\
\left(\mathrm{C}_{2} \mathrm{H}_{2} / \mathrm{C}_{2} \mathrm{H}_{4}\right)\end{array}$ & $\begin{array}{c}R_{2} \\
\left(\mathrm{CH}_{4} / \mathrm{H}_{2}\right)\end{array}$ & $\begin{array}{c}R_{3} \\
\left(\mathrm{C}_{2} \mathrm{H}_{4} / \mathrm{C}_{2} \mathrm{H}_{6}\right)\end{array}$ & Fault type \\
\hline$R_{1}>1.0$ & $0.1 \leq R_{2} \leq 0.5$ & $R_{3}>1.0$ & $\mathrm{D}_{1}$ \\
\hline $0.6 \leq R_{1} \leq 2.5$ & $0.1 \leq R_{2} \leq 1.0$ & $R_{3}>2.0$ & $\mathrm{D}_{2}$ \\
\hline$R_{1}<1.0$ & $R_{2}<0.1$ & $R_{3}<0.2$ & $\mathrm{PD}$ \\
\hline$R_{1}<1.0$ & $R_{2}>1.0$ & $R_{3}<1.0$ & $\mathrm{~T}_{1}$ \\
\hline$R_{1}<1.0$ & $R_{2}>1.0$ & $1.0 \leq R_{3} \leq 4.0$ & $\mathrm{~T}_{2}$ \\
\hline$R_{1}<1.0$ & $R_{2}>1.0$ & $R_{3}>4.0$ & $\mathrm{~T}_{3}$ \\
\hline
\end{tabular}

Table 3. Linguistic fault diagnosis based IEC60599 gas ratio limits.

\begin{tabular}{|c|c|c|c|}
\hline Gas ratio & IEC 60599 limits & Fault type case & Linguistic terms (L.T) \\
\hline \multirow{5}{*}{$\mathrm{C}_{2} \mathrm{H}_{2} / \mathrm{C}_{2} \mathrm{H}_{4}$} & $X_{1}<0.1$ & $\mathrm{PD}, \mathrm{T}_{1}, \mathrm{~T}_{2}, \mathrm{~T}_{3}$ & Very low (VL) \\
\hline & $0.1 \leq X_{1}<0.6$ & NS & Low (L) \\
\hline & $0.6 \leq X_{1} \leq 1.0$ & $\mathrm{D}_{2}$ & Medium (M) \\
\hline & $1.0<X_{1} \leq 2.5$ & $\mathrm{D}_{1}, \mathrm{D}_{2}$ & High $(\mathrm{H})$ \\
\hline & $X_{1}>2.5$ & $\mathrm{D}_{1}$ & Very High (VH) \\
\hline \multirow{5}{*}{$\mathrm{CH}_{4} / \mathrm{H}_{2}$} & $X_{2}<0.1$ & $\mathrm{PD}$ & Very low (VL) \\
\hline & $0.1 \leq X_{2} \leq 0.5$ & $\mathrm{D}_{1}, \mathrm{D}_{2}$ & Low (L) \\
\hline & $0.5<X_{2} \leq 1.0$ & $\mathrm{D}_{2}$ & Medium (M) \\
\hline & $1.0<X_{2} \leq 2.5$ & $\mathrm{~T}_{1}, \mathrm{~T}_{2}, \mathrm{~T}_{3}$ & $\operatorname{High}(\mathrm{H})$ \\
\hline & $X_{2}>2.5$ & $\mathrm{~T}_{1}, \mathrm{~T}_{2}, \mathrm{~T}_{3}$ & Very High (VH) \\
\hline \multirow{5}{*}{$\mathrm{C}_{2} \mathrm{H}_{4} / \mathrm{C}_{2} \mathrm{H}_{6}$} & $X_{3}<0.2$ & $\mathrm{PD}, \mathrm{T}_{1}$ & Very low (VL) \\
\hline & $0.2 \leq X_{3}<1.0$ & $\mathrm{~T}_{1}$ & Low (L) \\
\hline & $1.0<X_{3} \leq 2.0$ & $\mathrm{D}_{1}, \mathrm{~T}_{2}$ & Medium (M) \\
\hline & $2.0<X_{3} \leq 4.0$ & $\mathrm{D}_{1}, \mathrm{D}_{2}, \mathrm{~T}_{2}$ & High (H) \\
\hline & $X_{3}>4.04 .0$ & $\mathrm{D}_{1}, \mathrm{D}_{2}, \mathrm{~T}_{3}$ & Very High (VH) \\
\hline
\end{tabular}




\subsection{FRSN P Systems Fault Diagnosis}

FRSN P systems diagnostic model based DGA shown in Table 3, we can constrict the graphical diagnosis model of FRSN P systems with reasoning steps to identify the fault type of oil transformer, see Figure 3.

In this graphical model, IEC ratio with fuzzy representation as linguistic terms can built the FRSN P systems diagnostic model as shown in Figure 4.

Three ratio $R_{1}, R_{2}$ and $R_{3}$, each ratio with five levels very low $(\mathrm{VL})$, low $(\mathrm{L})$, medium $(\mathrm{M})$, high $(\mathrm{H})$ and very high( $\mathrm{VH})$ respectively as input proposition neuron with initial values and after reasoning steps, six fault types identified by confidence levels to give us which one with more confident with linguistic expression. This allowed us to diagnosis the fault with more informative and more correctly decisions.

From the historical database of transformer we can use the confidence level of each fault dissolved gas to use it in the matrix calculations of proposed method based on their experience operator and also we have to certainty factor to represent the degree of confidence fault occurs.

The rule neurons with synapse input neurons, the confidence $(0.8)$ and other rule neurons (1.0)

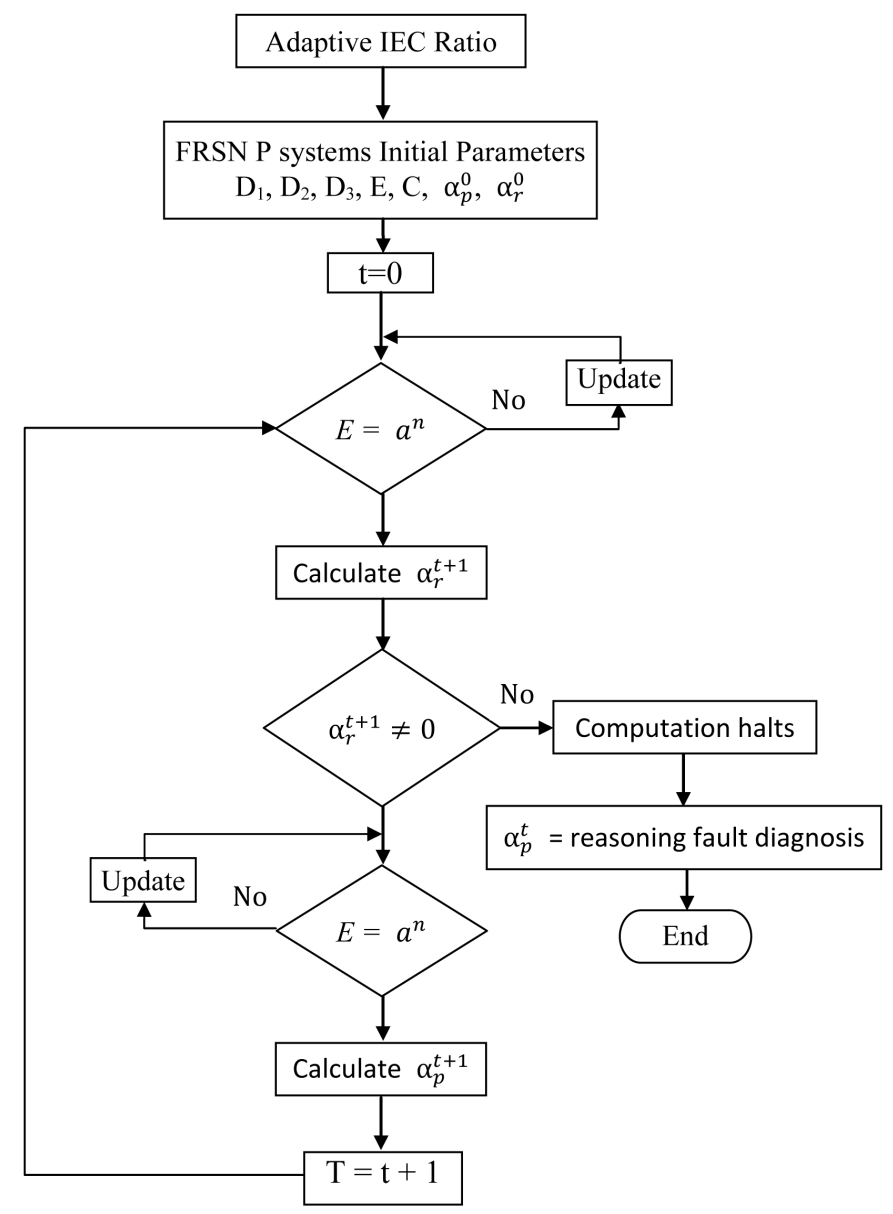

Figure 3. FRSN P systems transformer fault diagnosis flow chart. 


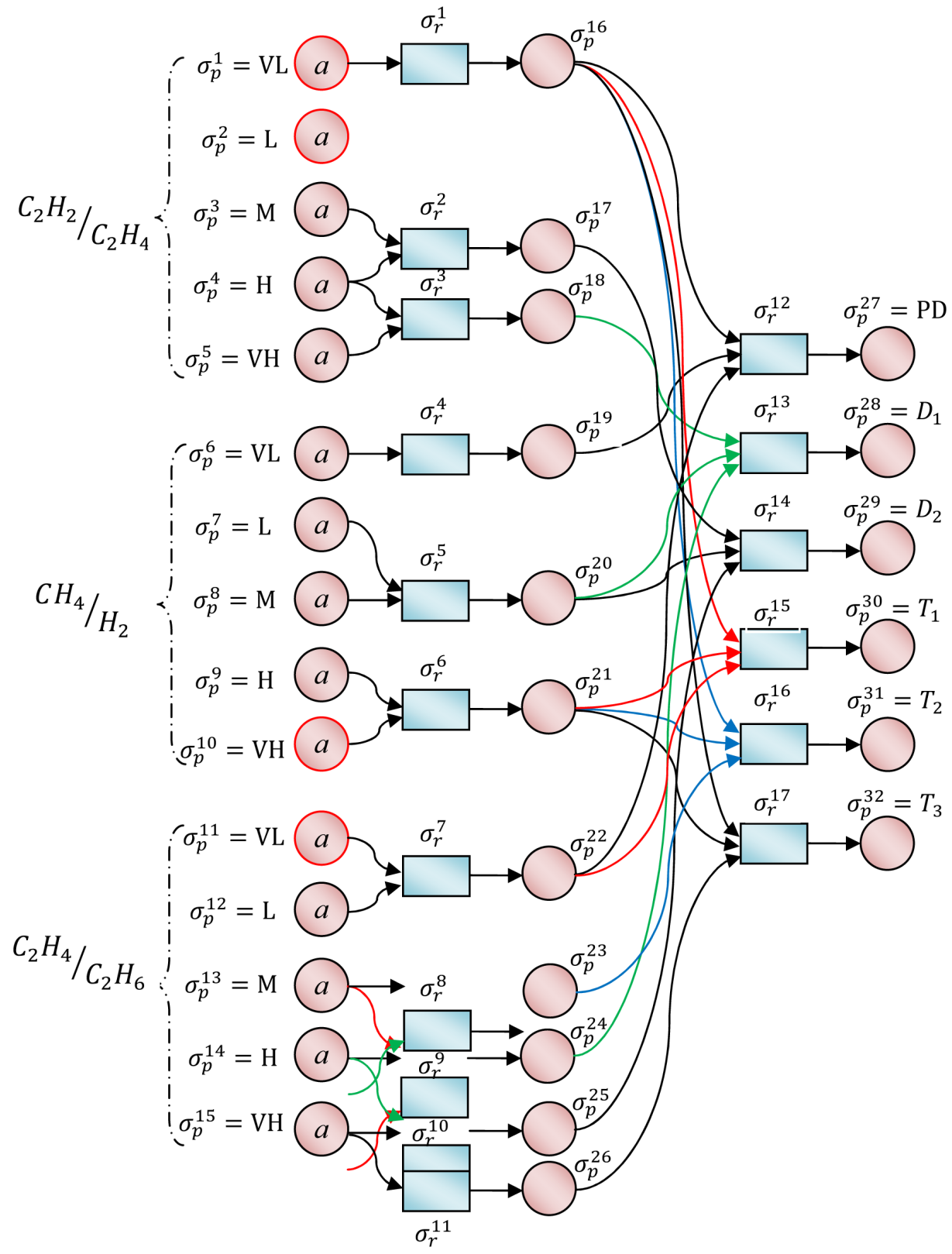

Figure 4. FRSN P systems fault diagnosis graphical model.

\subsection{Transformer Diagnosis Model Based on FRSN P Systems}

From the definition ( $\Pi$ ), we can use FRSN P systems to built fault diagnosis model for transformer based DGA ratio for all possible combinations of gases ratio based in AIEC ratio table, see Figure 4.

$$
\Pi=\left(A, \sigma_{1}, \cdots, \sigma_{m}, \text { syn }, I, O\right)
$$

$m=n+u, n=32$ proposition neurons, $u=17$ rule neurons

1. $A=\{a\}$ is the singleton alphabet (a is called spike);

2. $\sigma_{p}^{1}, \sigma_{p}^{2}, \cdots, \sigma_{p}^{32}$ are proposition neurons. 
3. $\sigma_{r}^{1}, \sigma_{r}^{2}, \cdots, \sigma_{r}^{17}$ are rule neurons;

where

a) $\sigma_{r}^{1}, \sigma_{r}^{4}$ and $\sigma_{r}^{11}$ are general rule neurons.

b) $\sigma_{r}^{2}, \sigma_{r}^{3}, \sigma_{r}^{5}, \cdots, \sigma_{r}^{10}$ are or rule neurons.

c) $\sigma_{r}^{12}, \cdots, \sigma_{r}^{17}$ are and rule neurons .

4. Syn. Shown in Figure 4.

5. in $=\left\{\sigma_{p}^{1}, \sigma_{p}^{2}, \cdots, \sigma_{p}^{15}\right\}$, out $=\left\{\sigma_{p}^{27}, \cdots, \sigma_{p}^{32}\right\}$.

\section{Testing, Results and Discussions}

This section presents the test cases of power transformer tested data to perform the proposed method, fuzzy reasoning Spiking Neural P systems (FRSN P systems) and evaluation with comparative with method with the same cases.

Table 4 shown the database of eight tested cases of gas transformer with different gas concentration of power transformers and use our proposed method to diagnosis the transformer with fault or no and classified as transformers with incipient faults and requires diagnosis.

From Tables 2-4 we can calculate the gas ratio and express by linguistic terms as shown in Table 5.

Table 4. Tested gas data of transformer.

\begin{tabular}{cccccc}
\hline No. & $\mathrm{H}_{2}$ & $\mathrm{CH}_{4}$ & $\mathrm{C}_{2} \mathrm{H}_{6}$ & $\mathrm{C}_{2} \mathrm{H}_{4}$ & $\mathrm{C}_{2} \mathrm{H}_{2}$ \\
\hline 1 & 19.3 & 103 & 159 & 19 & 0.6 \\
2 & 27 & 30 & 23 & 2.4 & 0.1 \\
3 & 23 & 63 & 54 & 10 & 0.3 \\
4 & 21 & 34 & 5 & 47 & 62 \\
5 & 160 & 130 & 33 & 96 & 0.1 \\
6 & 180 & 175 & 75 & 50 & 4 \\
7 & 345 & 112.3 & 27.5 & 51.5 & 58.8 \\
8 & 30.4 & 117 & 44.2 & 138 & 0.1 \\
\hline
\end{tabular}

Table 5. Ratio gas data with linguistic terms.

\begin{tabular}{ccccccc}
\hline \multirow{2}{*}{ No. } & \multicolumn{2}{c}{$\mathrm{C}_{2} \mathrm{H}_{2} / \mathrm{C}_{2} \mathrm{H}_{4}$} & \multicolumn{2}{c}{$\mathrm{CH}_{4} / \mathrm{H}_{2}$} & \multicolumn{2}{c}{$\mathrm{C}_{2} \mathrm{H}_{4} / \mathrm{C}_{2} \mathrm{H}_{6}$} \\
\cline { 2 - 7 } & ratio & L.T & ratio & L.T & ratio & L.T \\
\hline 1 & 0.03 & VL & 5.33 & VH & 0.12 & VL \\
2 & 0.04 & VL & 1.11 & H & 0.10 & VL \\
3 & 0.03 & VL & 2.74 & VH & 0.19 & VL \\
4 & 1.32 & H & 1.62 & H & 9.40 & VH \\
5 & 0.001 & VL & 0.813 & M & 2.909 & H \\
6 & 0.080 & VL & 0.972 & M & 0.666 & L \\
7 & 1.142 & H & 0.326 & L & 1.873 & M \\
8 & 0.0007 & VL & 3.849 & VH & 3.122 & H \\
\hline
\end{tabular}




\subsection{FRSN P Systems Diagnosis Matrix Reasoning Steps}

Each input proposition neurons will be assigned a truth degree value based on observation of the transformer history data, if the gas ratio limited values of AIEC ratio the of a transformer is actually observed, the input proposition neurons will have a truth degree value (0.9), otherwise truth degree value of non observed gases (0.1).

Each rule neurons with a certainty factor, which describes the confidence level based on experience of operator, in these cases, $c_{1}, c_{2}, \cdots, c_{11}$ will be given the same values (0.8) and $c_{12}, c_{13}, \cdots, c_{17}$ will be given the same values (1.0).

Case 1\#: The observed gases data are listed in Table 4 and gases ratio are listed in Table 5.

From $\Pi_{1}$ and Figure 4 , the input neurons $\sigma_{p}^{1}, \sigma_{p}^{2}, \cdots, \sigma_{p}^{15}$, the initial truth values of proposition neurons are $(0.9,0.1,0.1,0.1,0.1,0.1,0.1,0.1,0.1,0.9,0.9,0.1,0.1,0.1$, $0.1)$ respectively, and $\sigma_{p}^{16}, \sigma_{p}^{17}, \cdots, \sigma_{p}^{32}$ their truth values are (0), certainty factors corresponding to the rule neurons $\sigma_{r}^{1}, \sigma_{r}^{2}, \cdots, \sigma_{r}^{11}$ are given values (0.8) and $\sigma_{r}^{12}, \sigma_{r}^{13}, \cdots, \sigma_{r}^{17}(1.0)$.

The inference procedures are described step by step as follows:

$$
\theta_{p}^{0}=\left(\begin{array}{c}
(0.90) \\
(0.1)_{i=2,9} \\
(0.90) \\
(0.90) \\
(0.1)_{i=12, \cdots, 15} \\
(0)_{i=16, \cdots, 32}
\end{array}\right)_{32 \times 1}, \theta_{r}^{0}=(O)_{17 \times 1}
$$

where $\quad s_{i j}=1 \quad$ if $\quad(i, j)=\{(1,1),(6,4),(15,11)\} ; \quad$ otherwise, $\quad s_{i j}=0, \quad(1 \leq i \leq n$, $1 \leq j \leq u)$.

$$
S_{P \rightarrow \wedge}=\left(s_{i j}\right)_{32 \times 17}
$$

where $s_{i j}=1$ if $(i, j)=\{(16,12),(16,15),(16,16),(16,17),(17,14),(18,13),(19,12)$, $(20,13),(20,14),(21,15),(21,16),(21,17),(22,12),(22,15),(23,16),(24,13),(25,14)$, $(26,17)\}$; Otherwise, $s_{i j}=0,(1 \leq i \leq n, 1 \leq j \leq u)$.

$$
S_{P \rightarrow \vee}=\left(s_{i j}\right)_{32 \times 17}
$$

where $s_{i j}=1$ if $(i, j)=\{(3,2),(4,2),(4,3),(5,3),(7,5),(8,5),(9,6),(10,6),(11,7)$, $(12,7),(13,8),(13,9),(14,8),(14,9),(14,10),(15,9),(15,10)\}$; Otherwise, $s_{i j}=0$, $(1 \leq i \leq n, 1 \leq j \leq u)$.

$$
S_{R \rightarrow P}=\left(s_{j i}\right)_{32 \times 17}
$$

where $s_{j i}=1$ if $(j, i)=\{(1,16),(2,17),(3,18),(4,19),(5,20),(6,21),(7,22),(8,23)$, $(9,24),(10,25),(11,26),(12,27),(13,28),(14,29),(15,30),(16,31),(17,32)\}$; Otherwise, $s_{i j}=0,(1 \leq i \leq n, 1 \leq j \leq u)$.

$$
C=\operatorname{diag}\left(c_{1}, c_{2}, \cdots, c_{17}\right)
$$


where $c_{1}-c_{11}=(0.8), \quad c_{12}-c_{17}=(1.00)$.

At $t=0$

$$
\begin{aligned}
\theta_{r}^{1}=\left[S_{(P \rightarrow G)}^{T} \otimes \theta_{p}^{0}\right] & {\left[S_{(P \rightarrow \wedge)}^{T} \odot \theta_{p}^{0}\right]+\left[S_{(P \rightarrow \vee)}^{T} \circledast \theta_{p}^{0}\right] } \\
\theta_{r}^{1} & =\left(\begin{array}{c}
(0.9) \\
(0.1)_{i=2, \cdots, 5} \\
(0.9) \\
(0.9) \\
(0.1)_{i=8, \cdots, 11} \\
(0)_{j=12, \cdots, 17}
\end{array}\right)_{17 \times 1} \\
\theta_{p}^{1} & =S_{(R \rightarrow P)}^{T} \circledast\left[C \odot \theta_{r}^{1}\right] \\
\theta_{p}^{1} & =\left(\begin{array}{c}
(0)_{i=1, \cdots, 15} \\
(0.72) \\
(0.08)_{i=17, \cdots, 20} \\
(0.72) \\
(0.72) \\
(0.08)_{i=23, \cdots, 26} \\
(0)_{j=27, \cdots, 32}
\end{array}\right)_{32 \times 1}
\end{aligned}
$$

At $t=1$

$$
\begin{gathered}
\theta_{r}^{2}=\left[S_{(P \rightarrow G)}^{T} \otimes \theta_{p}^{1}\right]+\left[S_{(P \rightarrow \wedge)}^{T} \odot \theta_{p}^{1}\right]+\left[S_{(P \rightarrow \vee)}^{T} \circledast \theta_{p}^{1}\right] \\
\theta_{r}^{2}=\left(\begin{array}{c}
(0)_{i=1, \cdots, 11} \\
(0.08)_{i=12, \cdots, 14} \\
(0.72) \\
(0.08) \\
(0.08)
\end{array}\right)_{17 \times 1} \\
\theta_{p}^{2}=S_{(R \rightarrow P)}^{T} \circledast\left[C \odot \theta_{r}^{2}\right] \\
\theta_{p}^{2}=\left(\begin{array}{c}
(0)_{i=1, \cdots, 26} \\
(0.08)_{i=27, \cdots, 29} \\
(0.72) \\
(0.08) \\
(0.08)
\end{array}\right)_{32 \times 1}
\end{gathered}
$$

At $t=2$

$$
\begin{gathered}
\theta_{r}^{3}=\left[S_{(P \rightarrow G)}^{T} \otimes \theta_{p}^{2}\right]+\left[S_{(P \rightarrow \wedge)}^{T} \odot \theta_{p}^{2}\right]+\left[S_{(P \rightarrow \vee)}^{T} \circledast \theta_{p}^{2}\right] \\
\theta_{r}^{3}=(0)_{17 \times 1}
\end{gathered}
$$

Thus, the FRSN P system computation halts and the reasoning fault diagnosis results 
is $\theta_{p}^{2}=\left((0)_{i=1, \cdots, 26},(0.08)_{i=27, \cdots, 29}, 0.72,0.08,0.08\right)^{\mathrm{T}}$, The truth values of neurons propositions $\sigma_{p}^{27}, \sigma_{p}^{28}, \sigma_{p}^{29}, \sigma_{p}^{30}, \sigma_{p}^{31}$ and $\sigma_{p}^{32}$ are $0.08,0.08,0.08,0.72,0.08,0.08$

The reasoning results indicate the PD confidence $(0.08), \mathrm{D}_{1}$ confidence $(0.08), \mathrm{D}_{2}$ confidence (0.08), $\mathrm{T}_{1}$ confidence (0.72), $\mathrm{T}_{2}$ confidence $(0.08)$ and $\mathrm{T}_{3}$ confidence $(0.08)$.

So $\mathrm{T}_{1}$ with highest confidence level and greater than threshold $(0.50)$ is thermal faults $T<300^{\circ} \mathrm{C}$, and results for other cases are listed in Table 6 .

\subsection{Discussion Results}

In these cases, comparative studies of FRSN P systems with ratio support vector machine method (SVMR) and graphical support vector machine (SVMG), considered the same cases fault situations, the status tested gas data of transformer for eight tested cases are shown in Table 4, and the FRSN P systems diagnosis results are shown in Table 6. From the case studies $(1,2,3)$, the fault type is Thermal faults $T<300^{\circ} \mathrm{C}\left(\mathrm{T}_{1}\right)$ with confidence level $(0.72)$, case studies $(4,5,6)$ their isn't fault with confidence level $(0.08)$, case (7) is High energy discharge $\left(\mathrm{D}_{2}\right)$ and case (8) is Thermal faults $300<T<700^{\circ} \mathrm{C}$ $\left(T_{2}\right)$ fault with confidence level ( 0.72$)$.

Table 7 show us, the comparing results proposal method with SVMR and SVMG methods, according to test results in this table, the FRSN P systems is more suitable as dissolved gas signature and solved the problem of conflict between SVMR and SVMG.

\section{Conclusion}

In this study, the FRSN P systems technique has combined strength of uncertainty

Table 6. Ratio gas data with linguistic terms.

\begin{tabular}{cccc}
\hline \multirow{2}{*}{ Cases } & \multicolumn{2}{c}{ FRSN P systems Diagnosis Results } & \\
\cline { 2 - 4 } & Fault type & $\mathrm{CF}$ & Fault state \\
\hline \multirow{2}{*}{$\mathbf{1}$} & $\mathrm{D}_{1}, \mathrm{D}_{2}, \mathrm{PD}, \mathrm{T}_{2}, \mathrm{~T}_{3}$ & $(0.08)$ & No \\
& $\mathrm{T}_{1}$ & $(0.72)$ & Yes \\
& $\mathrm{D}_{1}, \mathrm{D}_{2}, \mathrm{PD}, \mathrm{T}_{2}, \mathrm{~T}_{3}$ & $(0.08)$ & No \\
$\mathbf{3}$ & $\mathrm{T}_{1}$ & $(0.72)$ & Yes \\
\multirow{2}{*}{$\mathbf{4}$} & $\mathrm{D}_{1}, \mathrm{D}_{2}, \mathrm{PD}, \mathrm{T}_{2}, \mathrm{~T}_{3}$ & $(0.08)$ & No \\
$\mathbf{5}$ & $\mathrm{T}_{1}$ & $(0.72)$ & Yes \\
$\mathbf{6}$ & $\mathrm{D}_{1}, \mathrm{D}_{2}, \mathrm{PD}, \mathrm{T}_{1}, \mathrm{~T}_{2}, \mathrm{~T}_{3}$ & $(0.08)$ & No \\
\multirow{2}{*}{$\mathbf{7}$} & $\mathrm{D}_{1}, \mathrm{D}_{2}, \mathrm{PD}, \mathrm{T}_{1}, \mathrm{~T}_{2}, \mathrm{~T}_{3}$ & $(0.08)$ & No \\
& $\mathrm{D}_{1}, \mathrm{D}_{2}, \mathrm{PD}, \mathrm{T}_{1}, \mathrm{~T}_{2}, \mathrm{~T}_{3}$ & $(0.08)$ & No \\
$\mathbf{8}$ & $\mathrm{D}_{1}, \mathrm{PD}, \mathrm{T}_{1}, \mathrm{~T}_{2}, \mathrm{~T}_{3}$ & $(0.08)$ & No \\
& $\mathrm{D}_{2}$ & $(0.72)$ & Yes \\
& $\mathrm{D}_{1}, \mathrm{D}_{2}, \mathrm{PD}, \mathrm{T}_{1}, \mathrm{~T}_{3}$ & $(0.08)$ & No \\
\hline
\end{tabular}


Table 7. Comparison FRSN P systems method with SVM method (SVMR/SVMG).

\begin{tabular}{cccc}
\hline \multirow{2}{*}{ Case No. } & \multicolumn{2}{c}{ SVM } & FRSN P systems \\
\cline { 2 - 3 } & SVMR & SVMG & $\mathrm{T}_{1}$ \\
\hline 1 & $\mathrm{~T}_{1}$ & $\mathrm{~T}_{2}$ & $\mathrm{~T}_{1}$ \\
3 & No fault & $\mathrm{T}_{1}$ & $\mathrm{~T}_{1}$ \\
4 & $\mathrm{~T}_{1}$ & $\mathrm{~T}_{2}$ & No fault \\
5 & No fault & $\mathrm{D}_{2}$ & No fault \\
6 & $\mathrm{~T}_{2}$ & $\mathrm{No} \mathrm{fault}_{2}$ & No fault \\
7 & $\mathrm{No} \mathrm{fault}_{8}$ & $\mathrm{~T}_{2}$ & $\mathrm{D}_{2}$ \\
$\mathrm{D}_{1}$ & $\mathrm{D}_{2}$ & $\mathrm{~T}_{2}$ \\
\hline
\end{tabular}

processing, rule-based reasoning, symbolic representation, and parallel computing. It makes transformer fault diagnosis based on DGA more accurate, fast and adaptive to system changes.

Especially, the reasoning process can be visualized in a form of graphical representation of FRSN P systems. The rule base and parameters are saved in matrix forms and the whole reasoning process is implemented by fuzzy matrix operations.

The aim of this study is to adaptive IEC Ratio with fuzzy representation and construct FRSN P systems diagnosis model to deal with fault transformers based on (IEC 60599) DGA as signature. Thus, the diagnosis model can be represent fuzzy production rules, dynamic reasoning algorithm and firing mechanism to diagnosis six types of fault transformer. Moreover, the practical test cases of transformer fault diagnosis are used to evaluate the proposed method.

This paper proposes FRSN P systems and tests its validity and feasibility in transformer fault diagnosis and comparing results with support vector machine (SVMR/SVMG) methods for the same fault cases.

Future work will focus on verifying the performance superiority of FRSN P systems, compared with other diagnosis methods; it can be integrated with other analysis applications comprehensive analysis.

\section{Acknowledgements}

This paper is partly supported by National Science Foundation of China (51577115).

\section{References}

[1] Bacha, K., Souahlia, S. and Gossa, M. (2012) Power Transformer Fault Diagnosis Based on Dissolved Gas Analysis by Support Vector Machine. Electric Power Systems Research, 83, 73-79. http://dx.doi.org/10.1016/j.epsr.2011.09.012

[2] Wang, T., Zhang, G.X., Zhao, J., He, Z., Wang, J. and Pérez-Jiménez, M.J. (2014) Fault Diagnosis of Electric Power Systems Based on Fuzzy Reasoning Spiking Neural P Systems. IEEE Transactions on Power Systems, 30, 1182-1194.

http://dx.doi.org/10.1109/TPWRS.2014.2347699 
[3] Fei, S.W. and Zhang, X.B. (2009) Fault Diagnosis of Power Transformer Based on Support Vector Machine with Genetic Algorithm. Expert Systems with Applications, 36, 11352 11357. http://dx.doi.org/10.1016/j.eswa.2009.03.022

[4] Liao, R.J., Zheng, H.B., Grzybowski, S., Yang, L.J., Tang, C. and Zhang, Y.Y. (2011) Fuzzy Information Granulated Particle Swarm Optimization Support Vector Machine Regression for the Trend Forecasting of Dissolved Gases in Oil-Filled Transformers. IET Electric Power Applications, 5, 230-237. http://dx.doi.org/10.1049/iet-epa.2010.0103

[5] Lv, G., Cheng, H., Zhai, H. and Dong, L. (2005) Fault Diagnosis of Power Transformer Based on Multi-Layer SVM Classifier. Electric Power Systems Research, 75, 9-15. http://dx.doi.org/10.1016/j.epsr.2004.07.013

[6] Yang, H.T. and Liao, C.C. (1999) Adaptive Fuzzy Diagnosis System for Dissolved Gas Analysis of Power Transformers. IEEE Transactions on Power Delivery, 14, 1342-1350. http://dx.doi.org/10.1109/61.796227

[7] Islam, M.S., Wu, T. and Ledwich, G. (2000) A Novel Fuzzy Logic Approach to Transformer Fault Diagnosis. IEEE Transactions on Dielectrics and Electrical Insulation, 7, 177-186. http://dx.doi.org/10.1109/94.841806

[8] Su, Q., Mi, C., Lai, L.L. and Austin, P. (2000) A Fuzzy Dissolved Gas Analysis Method for the Diagnosis of Multiple Incipient Faults in a Transformer. IEEE Transactions on Power Systems, 15, 593-598. http://dx.doi.org/10.1109/59.867146

[9] Afiqah, R.N., Musirin, I., Johari, D., Othman, M.M., Rahman, T.K.A. and Othman, Z. (2008) Fuzzy Logic Application in DGA Methods to Classify Fault Type in Power Transformer. Selected Topics in Power Systems and Remote Sensing, Malaysia.

[10] Liao, R., Zheng, H., Grzybowski, S., Yang, L., Zhang, Y. and Liao, Y. (2011) An Integrated Decision-Making Model for Condition Assessment of Power Transformers Using Fuzzy Approach and Evidential Reasoning. IEEE Transactions on Power Delivery, 26, 1111-1118. http://dx.doi.org/10.1109/TPWRD.2010.2096482

[11] Guardado, J.L., Naredo, J.L., Moreno, P., and Fuerte, C.R. (2001) A Comparative Study of Neural Network Efficiency in Power Transformers Diagnostic Using Dissolved Gas Analysis. IEEE Transactions on Power Delivery, 16, 643-647. http://dx.doi.org/10.1109/61.956751

[12] Hung, C.-P. and Wang, M.-H. (2004) Diagnosis of Incipient Faults in Power Transformers Using CMAC Neural Network Approach. Electric Power Systems Research, 71, 235-244. http://dx.doi.org/10.1016/j.epsr.2004.01.019

[13] Huang, Y.-C. (2003) Evolving Neural Nets for Fault Diagnosis of Power Transformer. IEEE Transactions on Power Delivery, 18, 843-848. http://dx.doi.org/10.1109/TPWRD.2003.813605

[14] Gunes, I., Gozutok, A., Ucan, O.N. and Kiremitci, B. (2009) Power Transformer Fault Type Estimation Using Artificial Neural Network Based on Dissolved Gas in Oil Analysis. International Journal of Engineering Intelligent System, 17, 193-198.

[15] Thukaram, D., Khincha, H.P. and Vijaynarasimha, H. (2005) Artificial Neural Network and Support Vector Machine Approach for Locating Faults in Radial Distribution Systems. IEEE Transactions on Power Delivery, 20, 710-721. http://dx.doi.org/10.1109/TPWRD.2005.844307

[16] Sun, Y.-J., Zhang, S., Miao, C.-X. and Li, J.-M. (2007) Improved BP Neural Network for Transformer Fault Diagnosis. Journal of China University of Mining \& Technology, 17, 138-142. http://dx.doi.org/10.1016/S1006-1266(07)60029-7

[17] Meng, K., Dong, Z.Y., Wang, D.H. and Wong, K.P. (2010) A Self-Adaptive RBF Neural Network Classifier for Transformer Fault Analysis. IEEE Transactions on Power Systems, 25, 1350-1360. http://dx.doi.org/10.1109/TPWRS.2010.2040491 
[18] Naresh, R., Sharma, V. and Vashisth, M. (2008) An Integrated Neural Fuzzy Approach for Fault Diagnosis of Transformers. IEEE Transactions on Power Delivery, 2017-2024. http://dx.doi.org/10.1109/TPWRD.2008.2002652

[19] Lin, C.H., Wu, C.H. and Huang, P.Z. (2009) Grey Clustering Analysis for Incipient Fault Diagnosis in Oil-Immersed Transformers. Expert Systems with Applications, 36, 13711379. http://dx.doi.org/10.1016/j.eswa.2007.11.019

[20] Lin, C.H., Chen, J.L. and Huang, P.Z. (2011) Dissolved Gases Forecast to Enhance OilImmersed Transformer Fault Diagnosis with Grey Prediction-Clustering Analysis. Expert Systems, 28, 123-137. http://dx.doi.org/10.1111/j.1468-0394.2010.00542.x

[21] Chen, W., Pan, C., Yun, Y. and Liu, Y. (2009) Wavelet Networks in Power Transformers Diagnosis Using Dissolved Gas Analysis. IEEE Transactions on Power Delivery, 24, 187 194. http://dx.doi.org/10.1109/TPWRD.2008.2002974

[22] Standard IEC 60599 (2007) Guide for the Interpretation of Dissolved Gas Analysis and GasFree.

[23] Peng, H., Wang, J., Pérez-Jiménez, M.J., Wang, H., Shao, J. and Wang, T. (2013) Fuzzy Reasoning Spiking Neural P System for Fault Diagnosis. Information Sciences, 235, 106116. http://dx.doi.org/10.1016/j.ins.2012.07.015

[24] Wang, J., Shi, P., Peng, H., Pérez-Jiménez, M.J. and Wang, T. (2013) Weighted Fuzzy Spiking Neural P System. IEEE Transactions on Fuzzy Systems, 21, 209-220. http://dx.doi.org/10.1109/TFUZZ.2012.2208974

[25] Wang, T., Zhang, G.X., Rong, H.N. and Perez-Jimenez, M.J. (2014) Application of Fuzzy Reasoning Spiking Neural P Systems to Fault Diagnosis. International Journal of Computers Communications \& Control, 9, 786-799. http://dx.doi.org/10.15837/ijccc.2014.6.1485

\section{Submit or recommend next manuscript to SCIRP and we will provide best service} for you:

Accepting pre-submission inquiries through Email, Facebook, LinkedIn, Twitter, etc. A wide selection of journals (inclusive of 9 subjects, more than 200 journals)

Providing 24-hour high-quality service

User-friendly online submission system

Fair and swift peer-review system

Efficient typesetting and proofreading procedure

Display of the result of downloads and visits, as well as the number of cited articles

Maximum dissemination of your research work

Submit your manuscript at: http://papersubmission.scirp.org/

Or contact jilsa@scirp.org 